\title{
Technology and business trends in regenerative medicine
}

\begin{abstract}
Tissue engineering and regenerative medicine gained industry interest about two decades ago. Since then, a number of therapies have received Food and Drug Administration clearance or approval and are commercially available, demonstrating successful results. Meanwhile, a comprehensive list of therapeutic strategies in preclinical and clinical investigation stages is continuously being expanded and explored in many regions around the world. Overall, most strategies are related to mimicking organ and tissue structures via scaffold fabrication, three-dimensional bioprinting, and self-assembly. Similarly, other approaches embrace the integration of grafts with the host via vascularization and innervation in order to provide broader and more accurate functionality. The induction of therapeutic responses by altering the host environment via cell infusion and immune system modulation has also gained special attention in the last five years. Especial focus has been directed to cell and gene therapies including cell-based immunotherapies induced pluripotent stem cells, tissue engineering and biomaterials, and small molecules and biologics.
\end{abstract}

In terms of business strategy, the health reform in Japan in 2015 changed the game rules, embracing multiple organizations and contract companies in space with the introduction of the new post-market surveillance strategy. As a result, Japan welcomes foreign companies for collaboration in multiple market sectors, impressively activating the economy around regenerative medicine.

Keywords: Allogeneic products, autologous products; Bioprinted products, cell banking, Cell reprogramming, cell therapy, Decellularized products, gene editing, Manufacturing workflow, regenerative medicine, stem cells
Volume 3 Issue 2 - 2017

\section{Cecilia Van Cauwenberghe}

Associate Fellow and Senior Industry Analyst in the TechVision Group at Frost \& Sullivan, USA

Correspondence: Cecilia Van Cauwenberghe,Associate Fellow and Senior Industry Analyst in the TechVision Group at Frost \& Sullivan, USA, Email cecilia.vancauwenberghe@frost.com

Received: November 07, 2017 | Published: November 29, 2017

\section{Regenerative medicine overall business pers- pective}

\section{Market challenges and opportunities}

The regenerative medicine market is heavily accelerated by the advent of stem cell technologies. Indeed, stem cells are attracting maximal attention in the regenerative medicine space. A growing number of companies have get into the market starting with the introduction of culture medium and reagents for regenerative medicine applications, to then advance toward the development of novel cellbased therapeutics based on stem cells. ${ }^{1}$ Induced pluripotent stem cells (iPSCs) are expected to significantly drive the market and advance stem cell therapeutics worldwide in the long-term. ${ }^{2}$

Frost \& Sullivan investigations valued the global market for regenerative medicine in 2016 in $\$ 30.1$ billion, with an expectation to rise to nearly $\$ 82.6$ billion by 2021 . As it is depicted in the Figure 1 , the compound annual growth rate (CAGR) constitutes $22.4 \%$ from the period 2016 to 2021 . $^{3}$

Political decisions, such as the Japanese reform of health laws, are dramatically leading business decisions re-shaping the medicine landscape. The case of Japan deserves special attention. The Japan's initiative that involved the reform of law based on regenerative medicine is attracting business toward the country. The creation of the Forum for Innovative Regenerative Medicine (FIRM) in 2014, comprising 185 firms as of January 2016, and the participation of an increasing number of organizations from the United States, Canada, the United Kingdom, Sweden, and Australia, represented a significant breakthrough. This fact derived in the health reform in Japan in 2015 with the new post-market surveillance strategy that enables practical use and market diffusion of regenerative medicine approaches.

The most remarkable challenges around regenerative medicine are related to the need for common standards in the development of devices, packaging, transportation, equipment, consumables, media and reagents, and services. Until 2018, these activities are expected to deeply impact the regenerative medicine space by consolidating several market niches. The transition to a dynamic market for innovation and entrepreneurship may proffera challenge for the medical industry.

Although regenerative medicine initiatives and programs involve the participation of strong stakeholders, both public funding and private investment, especially during research and developmental ways represent a critical point to be addressed. As Figure 2 illustrates, some business intelligence strategies need to be carried out in order to generate a more effective reimbursement that allows key players optimizing their investments by spending less efforts in clinical trials and more in therapeutics development.

\section{Technology transfer and clinical translation}

Breakthrough technologies are empowering regenerative medicine through the introduction of new, more accurate, easy-to-use tools. Hence, one of the most relevant innovations is related to the generation of autologous cells for transplantation. ${ }^{4}$ This technology demands the evolution of automatic monitoring and alarm systems to safeguard autologous cell transplants, driven by companies like co.don AG, Germany, through its Integrated Isolator technology (IIT). Similarly, next-generation patient- and donor-derived adoptive cellular therapies 
based on genetically modified $\mathrm{T}$ cells and natural killer (NK) cells require the application of novel cell engineering techniques and multigenic gene programs, such as those carried out by ZIOPHARM Oncology, US, Intrexon Corporation, US, The University of Texas MD Anderson Cancer Center, US, and Lonza Biologics, UK, in the development of the xeno-free platform L7TM System for the generation, maintenance, expansion, and cryopreservation of human induced pluripotent stem cells (iPSCs). Miltenyi Biotec, Germany, Cesca Therapeutics, US, Cytomatrix, Canada, Trans Tissue, Germany, and NantKwest, US, among many others, are hardly working in cell reprogramming, CAR-T cell technologies, bone marrow transplantation, blood expansion and immune cells transplantation. Major clinical translations are led by Asterias Biotherapeutics, US, with three clinical-stage development programs, Cartherics, Australia, with its dual targeting approach using iPSC technology to $\mathrm{T}$ cell receptor (TCR) and chimeric antigen receptor (CAR)-T engineered immune cells, and Organovo, US, with its breakthrough bioprinting technology on demand for research and medical applications.
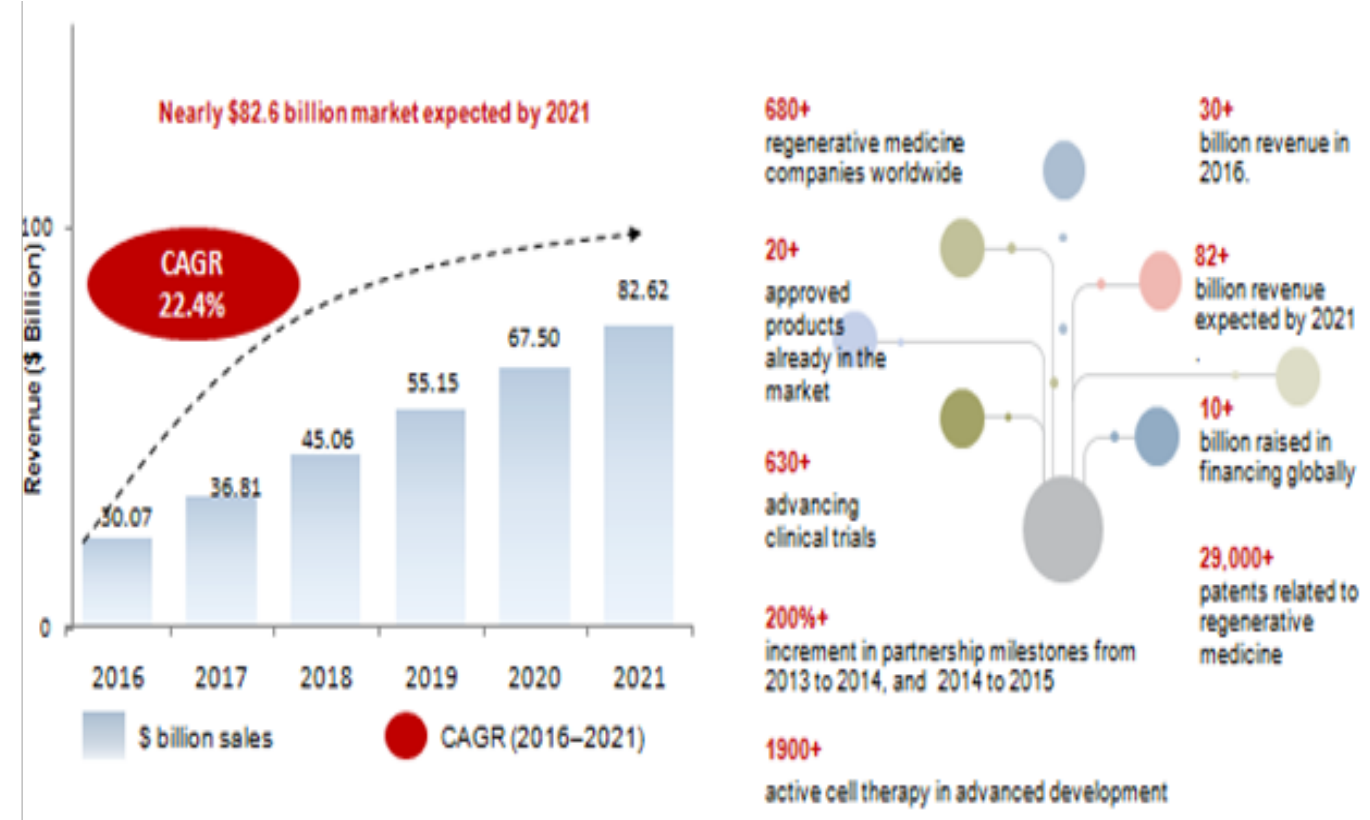

Figure I The global regenerative medicine market and principal facts, Forecast, 2016 to 202I. Source: Frost \& Sullivan.

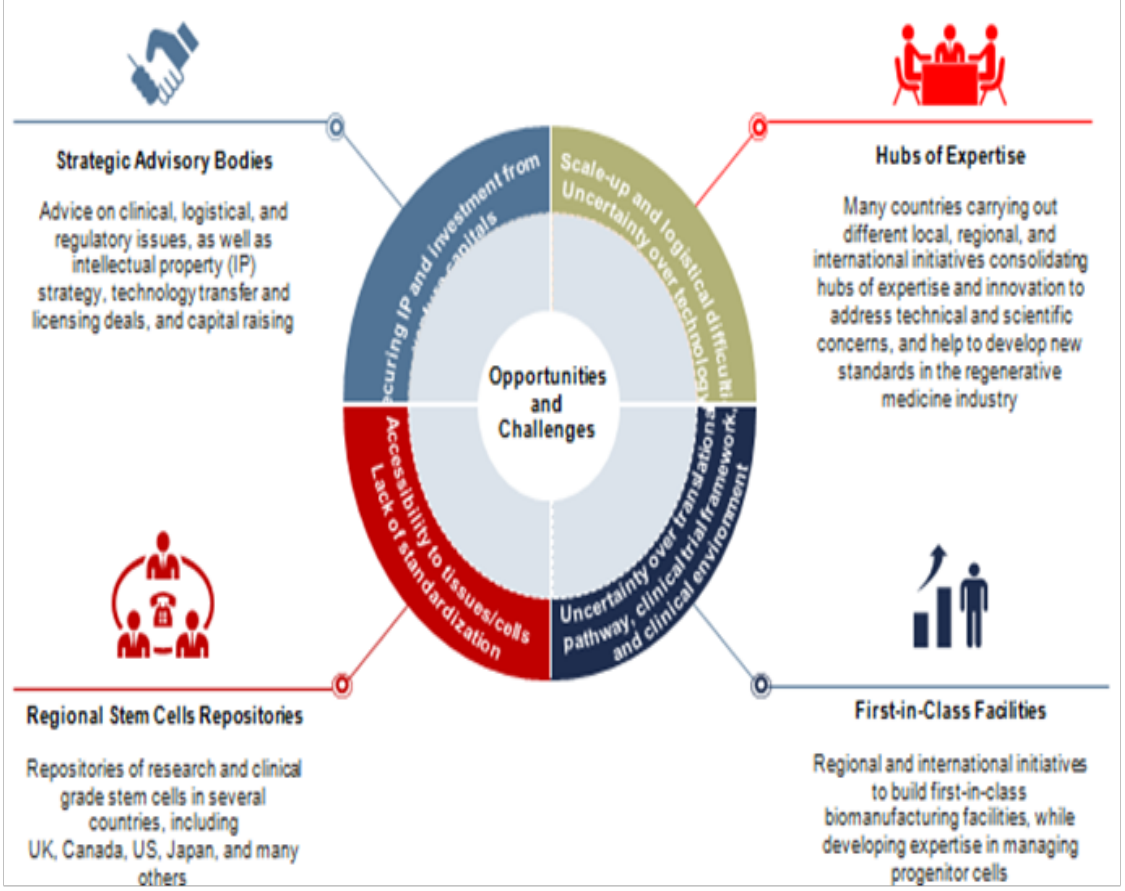

Figure 2 Technology/Business Trends: Prevalence of strategic advisory bodies. Source: Frost \& Sullivan. 


\section{Breakthroughs in manufacturing workflow strategies}

\section{Product manufacturing and workflow facilities}

According to the Japan's Ministry of Economy Trade and Industry (METI), allogeneic-based products are the most convenient for manufacturing scaling-up under on-demand production. Indeed, allogeneic production can be paralleled with the manufacturing workflows used in the production of biologics and medical devices. ${ }^{5}$
Nevertheless, unlike biopharmaceutical products, cells are living products and are carried forward through every processing stage. Thus, as it can be observed in the Figure 3, active monitoring of cellular yields and maintenance of the product's critical quality parameters, including purity, potency, and viability, is critical during the large-scale production run. Additional complexity is added when scaffolds are introduced. Quality attributes prior to seeding them with the therapeutic cell product needs to be guaranteed. Therefore, preconditioning, real-time monitoring, and noninvasive release testing procedures are strictly mandatory.

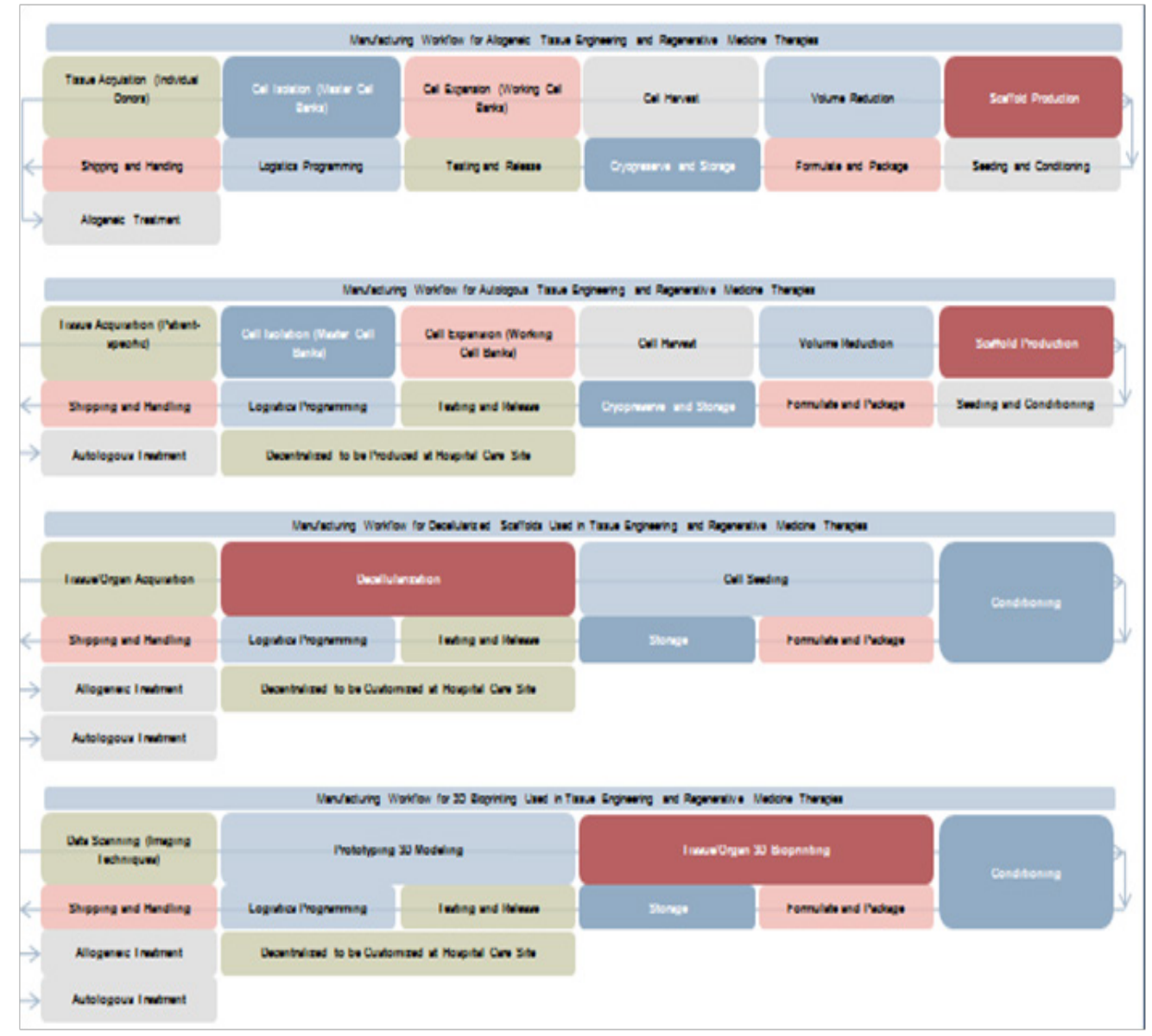

Figure 3 Regenerative medicine manufacturing workflow. Source: Frost \& Sullivan, Japan's ministry of economy trade and industry (METI).

Autologous-based products, on the other hand, are more suitable as patient-specific products, centered on the need for immunologic compatibility. Cells are obtained from individual donors, then expanded and processed following strict clinical specifications. Scaled-out approaches are simpler due to the possibility to run the parallel production of multiple, separate, and automated closed systems from multiple patients. Moreover, the same infrastructure is used repetitively. This demands that all the systems used at each processing stage need to be automated, closed, and tracked in real time involving single-use disposable equipment. As a result, distinct, parallel product flows with zero cross-contamination can be managed. When using scaffolds combined with the therapeutic cells, clinical specifications drive the structural architecture and mechanical characteristics according to the needs of a specific patient. In order to maintain product integrity and safety, process control and product testing at each stage constitute critical points. Preferable, shipping and the logistics of transporting a live product are simplified, for instance, by carrying out some production stages at the health institution. However, on site production still demands an in-depth understanding of autologous cell therapy at each stage of the process in order to deliver this service as an easy-to-use production platform that can be housed and set up within a healthcare institution.

Decellularized scaffolds are mostly used in tissue engineering and regenerative medicine therapies. Although their manufacturing workflow addresses a relatively less complex framework than cellbased approaches, the strategy must be designed carefully to avoid further issues. Broadly speaking, the manufacturing process involves the fabrication of an off-the-shelf product item, which can be modified on side within the clinic, where it is customized according to specific needs. Raw materials, which can be from both human and animal sources, may represent a challenge due to potential issues associated with contamination and disease transmission. Nevertheless, when decellularized, the scaffolds behave like any other medical device product, subjected to quality and safety regulations. 
Similarly, three-dimensional (3D) bioprinting includes the design, prototyping, and fabrication of 3D anatomical structures, mimicking organs, vasculature, and different types of tissues, such as skin, cartilage, bone, and connective tissue. ${ }^{6}$ The value chain model may involve both the production of an off-the-shelf product that can be used in many patients or a tailor-made product that address individual medical needs. An alternative for this latter option relies on the decentralization of the activities, by housing a 3D bioprinter in the health institution. This type of real-time biofabrication of the product could even be adapted into the surgical suite.

\section{World class-accredited manufacturing investments}

The past two years have showed substantial investment in world class-accredited manufacturing facilities for regenerative medicine approaches, especially regarding immunocellular therapy. Just to mention some examples, in the United States, Kite Pharma launched a new production plant in California; Adapt Immune and $\mathrm{WuXi}$ AppTec are heavily investing in leasing manufacturing facilities in Philadelphia; Lonza is expanding manufacturing capabilities in Houston; UniQure built the largest gene therapy production facility in Massachusetts. Comparably, Green Cross is expanding cell therapy facilities and operations in China. All these facts place the regenerative medicine field in a very active position in terms of the creation of new companies around a highly diversified spectrum of products and services. Correspondingly, the regenerative medicine field constitutes one of the most intensive sources of highly skilled employment in the healthcare sector. Supporting this statement, Cell Therapy Catapult and Mayo Clinic are hosting multiple commercial cell therapy developers in a period of five years, hence fueling the regional economy. Many other large pharmaceutical and biotechnology companies are shifting from contracting and outsourcing to in-house manufacturing. This fact also re-shapes the space in terms of bio manufacturing capabilities.

Regarding public funding, a sustained annual average of $\$ 2.5$ million is exhibited by National Institute of Standards and Technology (NIST) during the period 2012-2016. The National Institute of Health (NIH) is primarily involved in basic, translational, and clinical research, which is demonstrated through near 2,400 awards per year during the same period, especially associated with the National Heart, Lung, and Blood Institute, National Institute of Diabetes and Digestive and Kidney Diseases, National Cancer Institute, and National Institute of Neurological Disorders and Stroke. Each of them has been funded over \$200 million.

\section{Conclusion and final remarks}

Regenerative medicine constitutes one of the most affluent fields of research boosting the creation of new companies offering both products and services within a vast range of logical innovations, from autologous cell transplantation in the clinics to bioprinted tissue for research use and validation tests in pharmaceutical development. Novel therapies using regenerative medicine approaches are expected to be successfully translated to the clinic during the coming five years. The advent of stem cell delivery platforms for the treatment of a variety of life threatening diseases is motivating companies to attend this niche. Equivalent trends in biotechnology, including predictive biomarkers, along with the development of smart materials and flexible electronics paved the way of the development of 3D bioplotting scaffolds for the improvement of cells transplantation.

The regenerative medicine market is remarkably displaying those investigational drifts with the introduction of new therapeutic products and smart devices currently undergoing clinical validation. In a research service published by Frost et al., ${ }^{8}$ an analytical hierarchy process (AHP) tool is utilized to assess the impact of multiple factors affecting regenerative medicine business performance. Figure 4 exhibits the criteria used in the analysis. As part of the analysis, the experts found that the regenerative medicine market landscape is expected to evidence a great revolution in terms of products launched and customer niches penetrated in the coming five years. Therefore, costs associated with cycle developmental time around novel solutions become decisive for commercialization success. Operations must be commenced promptly allowing recovering the investment made during development processes. Consequently, fast-to-market strategies along with the post-market surveillance approach pioneered by Japan will significantly pull the market.

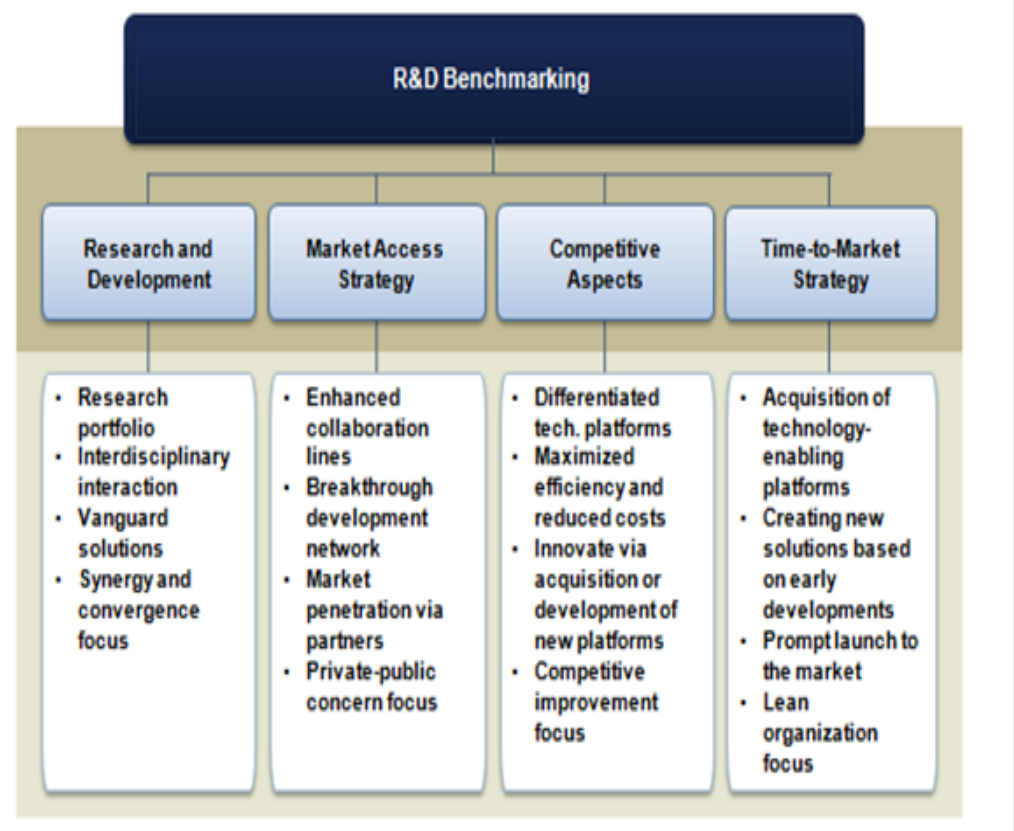

Figure 4 Regenerative medicine principal business and technology strategies. Source: Frost \& Sullivan. 
Still demanding more expertise and business modeling, insurance coverage and reimbursement strategies are critical factors to promote the clinical translation of regenerative medicine technologies.

Therefore, regional expansion and collaboration networks will sustainable gaining attention over the next five years period. Licensing deals are becoming crucial for market positioning through the achievement of common goals and earlier delivery of regenerative medicine products within the market. Acquisition of companies or development of proprietary regenerative medicine platforms presently constitutes the most effective decision to advance the regenerative medicine pipeline. Beyond funding and investment needs, strong focus must be directed to the development of new innovation attributes as a result of the synergy among leading-edge technologies in order to remark a competitive aspect.

\section{Acknowledgements}

I would like to thank all contributors from industry involved with the development and delivery of this article and Frost \& Sullivan's staff from the TechVision Group.

\section{Conflict of interest}

The author declares no conflict of interest.

\section{References}

1. Wobma H, Vunjak Novakovic G. Tissue engineering and regenerative medicine 2015: a year in review. Tissue Eng Part B Rev. 2016;22(2):101113.

2. Revilla A, González C, Iriondo A, et al. Current advances in the generation of human iPS cells: implications in cell-based regenerative medicine. J Tissue Eng Regen Med. 2016;10(11):893-907.

3. Van Cauwenberghe C. Regenerative medicine: How stem cell therapy is innovating healthcare. Frost \& Sullivan Market Insight, USA: Springer; 2016.

4. Khang G. Handbook of intelligent scaffolds for tissue engineering and regenerative medicine. USA: CRC Press; 2017. 974 p.

5. Groll J, Boland T, Blunk T, et al. Biofabrication: reappraising the definition of an evolving field. Biofabrication. 2016;8(1):013001.

6. Gaharwar AK, Arpanaei A, Andresen TL, et al. 3D biomaterial microarrays for regenerative medicine: current state-of-the-art, emerging directions and future trends. Adv Mater. 2016;28(4):771-781.

7. Gao G, Cui X. Three-dimensional bioprinting in tissue engineering and regenerative medicine. Biotechnol Lett. 2016;38(2):203-211.

8. Van Cauwenberghe C. Regenerative Medicine-The Future of Therapy (TechVision), Re-shaping Global Healthcare. Frost \& Sullivan Research Service D6EC-01, USA: Springer; 2016. 